\title{
Asymptotic stability of solutions to a class of linear time-delay systems with periodic coefficients and a large parameter
}

\author{
Gennadii V Demidenko ${ }^{1,2^{*}}$ and Inessa I Matveeva ${ }^{1,2}$
}

"Correspondence:

demidenk@math.nsc.ru

${ }^{1}$ Laboratory of Differential and

Difference Equations, Sobolev

Institute of Mathematics, Acad.

Koptyug avenue 4, Novosibirsk,

630090, Russia

${ }^{2}$ Department of Mechanics and

Mathematics, Novosibirsk State

University, Pirogov str. 2,

Novosibirsk, 630090, Russia

\begin{abstract}
A class of linear time-delay systems with periodic coefficients and a large parameter is studied. We establish conditions under which the zero solution is asymptotically stable. This result allows us to study the asymptotic stability of the zero solution to the time-delay systems without spectral methods and Lyapunov-Krasovskii functionals.
\end{abstract}

MSC: Primary 34K20; secondary 34D20

Keywords: time-delay equations; periodic coefficients; asymptotic stability; Lyapunov differential equation; Riccati type matrix differential inequality

\section{Introduction}

There are a large number of works devoted to delay differential equations (for instance, see [1-11] and the bibliography therein). One of the interesting questions is the asymptotic stability of solutions to delay differential equations. This question is very important from theoretical and practical viewpoints, because delay differential equations arise in many applied problems when describing the processes whose rates of change are defined by present and previous states (for example, see [12-14] and the bibliography therein). The case of variable coefficients is of special interest and is more complicated in comparison with the case of constant coefficients.

This article presents a continuation of our work on stability of solutions to delay differential equations. We consider linear systems of delay differential equations with periodic coefficients of the form

$$
\frac{d}{d t} y(t)=\mu A(t) y(t)+B(t) y(t-\tau), \quad t>0,
$$

where $A(t), B(t)$ are $(n \times n)$ matrices with $T$-periodic continuous entries; i.e.,

$$
A(t+T) \equiv A(t), \quad B(t+T) \equiv B(t)
$$

$\tau>0$ is the time delay, and $\mu>0$ is a parameter. We suppose that the spectrum of the matrix $A(t)$ belongs to the left half-plane $\mathbb{C}_{-}=\{\lambda \in \mathbb{C}: \operatorname{Re} \lambda<0\}$ for every $t \geq 0$. Using our results of [15-17], we prove that in this case there exists $\mu_{*}>0$ such that the zero solution to (1) is asymptotically stable for $\mu>\mu_{*}$ and suggest a way for calculating $\mu_{*}$.

(c) 2015 Demidenko and Matveeva. This article is distributed under the terms of the Creative Commons Attribution 4.0 International License (http://creativecommons.org/licenses/by/4.0/), which permits unrestricted use, distribution, and reproduction in any medium, provided you give appropriate credit to the original author(s) and the source, provide a link to the Creative Commons license, and indicate if changes were made. 


\section{Preliminaries}

In [15-20] we studied the question about the asymptotic stability of solutions to systems of ordinary differential equations and systems of delay differential equations with periodic coefficients. We proved theorems on asymptotic stability which are analogs of the classic theorems on the asymptotic stability for equations with constant coefficients. We now formulate two theorems used hereinafter.

First, we recall the criterion [15] for the asymptotic stability of the zero solution to the system of ordinary differential equations with $T$-periodic coefficients

$$
\frac{d}{d t} y(t)=A(t) y(t), \quad A(t+T) \equiv A(t) .
$$

Hereinafter the matrix inequality $Q>0$ (or $Q<0$ ) means that the matrix $Q$ is positive (or negative) definite.

Theorem 1 I. If the zero solution to (2) is asymptotically stable, then, for every continuous matrix $C(t)$ on $[0, T]$, there is a unique solution $H(t)$ to the boundary value problem

$$
\left\{\begin{array}{l}
\frac{d}{d t} H+H A(t)+A^{*}(t) H=-C(t), \quad 0<t<T, \\
H(0)=H(T)
\end{array}\right.
$$

moreover, if

$$
C(t)=C^{*}(t)>0, \quad t \in[0, T]
$$

then

$$
H(t)=H^{*}(t)>0, \quad t \in[0, T] .
$$

II. Suppose that the right-hand side $C(t)$ of the Lyapunov differential equation is continuous on $[0, T]$ and satisfies (4). If the boundary value problem (3) has a Hermitian solution $H(t)$ such that $H(0)>0$, then the zero solution to (2) is asymptotically stable.

It should be noted that, in the case of constant coefficients, this result coincides with the Lyapunov criterion. Using Theorem 1 and the Lyapunov-Krasovskii functionals introduced in [16], we proved the following theorem $[16,17]$ on the asymptotic stability of the zero solution to the time-delay system

$$
\frac{d}{d t} y(t)=A(t) y(t)+B(t) y(t-\tau), \quad t>0,
$$

where

$$
A(t+T) \equiv A(t), \quad B(t+T) \equiv B(t) .
$$

Theorem 2 Suppose that there are matrices

$$
L(t)=L^{*}(t) \in C^{1}[0, T] \quad \text { and } \quad K(s)=K^{*}(s) \in C^{1}[0, \tau]
$$


such that

$$
L(0)=L(T)>0, \quad K(s)>0, \quad \frac{d}{d s} K(s)<0, \quad s \in[0, \tau],
$$

and the following Riccati type matrix differential inequality holds:

$$
\frac{d}{d t} L(t)+L(t) A(t)+A^{*}(t) L(t)+L(t) B(t) K^{-1}(\tau) B^{*}(t) L(t)+K(0)<0
$$

for $t \in[0, T]$. Then the zero solution to (5) is asymptotically stable.

Using the mentioned matrices $L(t), K(s)$, estimates characterizing exponential decay of solutions to (5) at infinity were established in $[16,17]$.

Before studying the asymptotic stability of the zero solution to (1), firstly we consider the system of ordinary differential equations of the form

$$
\frac{d}{d t} y(t)=\mu A(t) y(t), \quad t>0, \quad A(t+T) \equiv A(t) .
$$

Let the spectrum of $A(t)$ belong to the left half-plane $\mathbb{C}_{-}$for every $t \geq 0$ and $\mu>0$. It is well known that there are examples of periodic matrices $A(t)$ such that the zero solution to (9) is not asymptotically stable for arbitrary $\mu>0$. Indeed, consider (see [21]) the system (9) with

$$
A(t)=\left(\begin{array}{cc}
-2 \cos (4 t)-1 & 2 \sin (4 t)+2 \\
2 \sin (4 t)-2 & 2 \cos (4 t)-1
\end{array}\right)
$$

and $\mu=1$. Obviously, $\lambda_{1}=-1, \lambda_{2}=-1$ are the eigenvalues of $A(t)$. It is not hard to verify that

$$
y(t)=\left(\begin{array}{l}
y_{1}(t) \\
y_{2}(t)
\end{array}\right)=\left(\begin{array}{l}
e^{t} \sin (2 t) \\
e^{t} \cos (2 t)
\end{array}\right)
$$

is a solution to (9) for $\mu=1$. Clearly,

$$
\|y(t)\| \rightarrow \infty, \quad t \rightarrow \infty
$$

and the zero solution to this system is not stable (here $\|y(t)\|=\sqrt{y_{1}^{2}(t)+y_{2}^{2}(t)}$ ). This example shows that the zero solution to (9) is not asymptotically stable for arbitrary $\mu>0$. At the same time, according to Krein's result, the zero solution to (9) is asymptotically stable for $\mu \gg 0$ large enough (see [22]). Below we propose a way for calculating a value $\mu_{0}>0$ such that the zero solution to (9) is asymptotically stable for $\mu>\mu_{0}$. This result will be used in the next section.

Since the spectrum of $A(t)$ belongs to the left half-plane $\mathbb{C}_{-}$for every $t \in[0, T]$, by the Lyapunov criterion, for every fixed $t \in[0, T]$, there exists a unique solution $H(t)=H^{*}(t)>$ 0 to the Lyapunov matrix equation

$$
H A(t)+A^{*}(t) H=-I .
$$


Introduce the following notations: $H_{\max }=\max _{t \in[0, T]}\|H(t)\|, v_{\max }=\max _{t \in[0, T]} v(H(t))$, where $v(H(t))=\|H(t)\|\left\|H^{-1}(t)\right\|$ is the condition number of $H(t)$. Here and hereafter we use the spectral norm of matrices.

Theorem 3 Let $N$ be a number such that the following inequality holds:

$$
\max _{|t-s| \leq T}\|A(t)-A(s)\| \leq \frac{1}{4 H_{\max } \sqrt{v_{\max }}} .
$$

Then the zero solution to (9) is asymptotically stable for

$$
\mu>\mu_{0}=\frac{2 N H_{\max }}{T} \ln v_{\max }
$$

Proof Let $Y(t)$ be the matriciant of (9); i.e., $Y(t)$ is a solution to the Cauchy problem

$$
\left\{\begin{array}{l}
\frac{d}{d t} Y=\mu A(t) Y, \quad t>0 \\
\left.Y\right|_{t=0}=I
\end{array}\right.
$$

Obviously, for every fixed $t_{j} \geq 0$, the matrix $Y(t)$ is a solution to the Cauchy problem

$$
\left\{\begin{array}{l}
\frac{d}{d t} Y=\mu A(t) Y, \quad t>t_{j} \geq 0 \\
\left.Y\right|_{t=t_{j}}=Y_{j}
\end{array}\right.
$$

where $Y_{j}=Y\left(t_{j}\right)$. Rewrite this Cauchy problem in the form

$$
\left\{\begin{array}{l}
\frac{d}{d t} Y=\mu A\left(t_{j}\right) Y+\mu\left(A(t)-A\left(t_{j}\right)\right) Y, \quad t>t_{j} \geq 0, \\
\left.Y\right|_{t=t_{j}}=Y_{j} .
\end{array}\right.
$$

Clearly, every solution to the problem is a solution to the integral matrix equation

$$
Y(t)=\exp \left(\left(t-t_{j}\right) \mu A\left(t_{j}\right)\right) Y_{j}+\int_{t_{j}}^{t} \exp \left((t-s) \mu A\left(t_{j}\right)\right) \mu\left(A(s)-A\left(t_{j}\right)\right) Y(s) d s .
$$

Consequently,

$$
\begin{aligned}
\|Y(t)\| \leq & \left\|\exp \left(\left(t-t_{j}\right) \mu A\left(t_{j}\right)\right)\right\|\left\|Y_{j}\right\| \\
& +\mu \int_{t_{j}}^{t}\left\|\exp \left((t-s) \mu A\left(t_{j}\right)\right)\right\|\left\|A(s)-A\left(t_{j}\right)\right\|\|Y(s)\| d s .
\end{aligned}
$$

Since the spectrum of $A\left(t_{j}\right)$ belongs to the left half-plane $\mathbb{C}_{-}$, by Krein's inequality [22] we have

$$
\left\|\exp \left(t A\left(t_{j}\right)\right)\right\| \leq \sqrt{v\left(H_{j}\right)} \exp \left(-\frac{t}{2\left\|H_{j}\right\|}\right), \quad t>0,
$$

where $H_{j}$ is a solution to the Lyapunov matrix equation

$$
H_{j} A\left(t_{j}\right)+A^{*}\left(t_{j}\right) H_{j}=-I,
$$


$v\left(H_{j}\right)=\left\|H_{j}\right\|\left\|H_{j}^{-1}\right\|$. Using (12), from (11) we obtain

$$
\begin{aligned}
\|Y(t)\| \leq & \sqrt{v\left(H_{j}\right)} \exp \left(-\frac{\left(t-t_{j}\right) \mu}{2\left\|H_{j}\right\|}\right)\left\|Y_{j}\right\| \\
& +\mu \sqrt{v\left(H_{j}\right)} \int_{t_{j}}^{t} \exp \left(-\frac{(t-s) \mu}{2\left\|H_{j}\right\|}\right)\left\|A(s)-A\left(t_{j}\right)\right\|\|Y(s)\| d s .
\end{aligned}
$$

According to the conditions of the theorem, if $t \in\left[t_{j}, t_{j}+\frac{T}{N}\right]$ then

$$
\left\|A(t)-A\left(t_{j}\right)\right\| \leq \frac{1}{4 H_{\max } \sqrt{\nu_{\max }}} .
$$

Hence,

$$
\|Y(t)\| \leq \sqrt{v_{\max }} \exp \left(-\frac{\left(t-t_{j}\right) \mu}{2 H_{\max }}\right)\left\|Y_{j}\right\|+\frac{\mu}{4 H_{\max }} \int_{t_{j}}^{t} \exp \left(-\frac{(t-s) \mu}{2 H_{\max }}\right)\|Y(s)\| d s .
$$

From Gronwall's inequality (for example, see [23]) we have

$$
\|Y(t)\| \leq \sqrt{\nu_{\max }}\left\|Y_{j}\right\| \exp \left(-\frac{\left(t-t_{j}\right) \mu}{4 H_{\max }}\right), \quad t \in\left[t_{j}, t_{j}+\frac{T}{N}\right]
$$

Let $t_{j}=\frac{j T}{N}, j=0, \ldots, N$. By (13) we obtain

$$
\left\|Y_{j}\right\| \leq \sqrt{\nu_{\max }}\left\|Y_{j-1}\right\| \exp \left(-\frac{T \mu}{4 N H_{\max }}\right), \quad j=1, \ldots, N
$$

Since $\left\|Y_{0}\right\|=\|Y(0)\|=1$,

$$
\left\|Y_{j}\right\| \leq\left(\sqrt{\nu_{\max }}\right)^{j} \exp \left(-\frac{j T \mu}{4 N H_{\max }}\right), \quad j=1, \ldots, N
$$

Consequently, from (13) we derive

$$
\|Y(t)\| \leq\left(\sqrt{v_{\max }}\right)^{j+1} \exp \left(-\frac{t \mu}{4 H_{\max }}\right), \quad t \in\left[t_{j}, t_{j+1}\right], j=0, \ldots, N-1 .
$$

As a result, we have the following estimate for the matriciant of (9):

$$
\|Y(t)\| \leq\left(\sqrt{v_{\max }}\right)^{N} \exp \left(-\frac{t \mu}{4 H_{\max }}\right), \quad t \in[0, T] .
$$

Then the following inequality holds for the monodromy matrix:

$$
\|Y(T)\| \leq\left(\sqrt{v_{\max }}\right)^{N} \exp \left(-\frac{\mu T}{4 H_{\max }}\right)
$$

Hence, if $\mu$ satisfies (10) then $\|Y(T)\|<1$ and the spectrum of the monodromy matrix $Y(T)$ belongs to the unit disk $\{\lambda \in \mathbb{C}:|\lambda|<1\}$. Consequently, in view of the spectral criterion, the zero solution to (9) is asymptotically stable.

The theorem is proved. 
Remark 1 It should be noted that $N$ satisfying the conditions of Theorem 3 exists owing to the continuity of the entries of $A(t)$ on $[0, T]$.

Remark 2 It follows from the proof that all solutions to (9) tend to zero as $t \rightarrow \infty$.

\section{Asymptotic stability of solutions to delay differential equations}

We now consider the time-delay systems of the form (1). Using the theorems formulated in Section 2 , we indicate a value $\mu_{*}$ such that the zero solution to (1) is asymptotically stable for $\mu>\mu_{*}$.

Theorem 4 Let $N$ be a number such that the following inequality holds:

$$
\max _{|t-s| \leq \frac{T}{N}}\|A(t)-A(s)\| \leq \frac{1}{4 H_{\max } \sqrt{v_{\max }}}
$$

let $\mu_{*}>0$ satisfy the equality

$$
\mu_{*}\left(1-\left(v_{\max }\right)^{N} \exp \left(-\frac{\mu_{*} T}{2 H_{\max }}\right)\right)=4 e^{\alpha \tau / 2} H_{\max }\left(v_{\max }\right)^{N} \max _{t \in[0, T]}\|B(t)\|
$$

for some $\alpha>0$. Then the zero solution to (1) is asymptotically stable for $\mu>\mu_{*}$.

Proof By Theorem 2, if there are matrices $L(t)$ and $K(s)$ satisfying (6), (7), and the Riccati type matrix differential inequality of the form $(8)$

$$
\frac{d}{d t} L(t)+\mu L(t) A(t)+\mu A^{*}(t) L(t)+L(t) B(t) K^{-1}(\tau) B^{*}(t) L(t)+K(0)<0,
$$

then the zero solution to (1) is asymptotically stable. Let us show that such matrices $L(t)$ and $K(s)$ exist.

It is not hard to see that $\mu_{*}>\mu_{0}$, where $\mu_{0}$ is defined in (10). Consequently, by Theorem 3 the zero solution to (9) is asymptotically stable for $\mu>\mu_{*}$; moreover, (14) is satisfied. Hence, according to Theorem 1, for such $\mu$ there exists a unique solution $L(t)=L^{*}(t)$ to the boundary value problem

$$
\left\{\begin{array}{l}
\frac{d}{d t} L+\mu L A(t)+\mu A^{*}(t) L=-I, \quad 0<t<T, \\
L(0)=L(T)>0 .
\end{array}\right.
$$

Using the matriciant $Y(t)$ of (9), the solution to this problem can be written in the form (see [15])

$$
\begin{aligned}
L(t) & =\left(Y^{-1}(t)\right)^{*}\left[\int_{t}^{\infty}(Y(s))^{*} Y(s) d s\right] Y^{-1}(t) \\
& =\int_{0}^{\infty}\left(Y(\eta+t) Y^{-1}(t)\right)^{*}\left(Y(\eta+t) Y^{-1}(t)\right) d \eta .
\end{aligned}
$$

Consider the matrix

$$
\tilde{Y}(\eta)=Y(\eta+t) Y^{-1}(t)
$$


for every fixed $t$. Obviously, it is the matriciant of the system

$$
\frac{d}{d \eta} \tilde{y}(\eta)=\mu A(\eta+t) \tilde{y}(\eta)
$$

By (14), we have the estimate

$$
\|\tilde{Y}(\eta)\| \leq\left(\sqrt{v_{\max }}\right)^{N} \exp \left(-\frac{\mu \eta}{4 H_{\max }}\right), \quad \eta \in[0, T]
$$

Using the property of the matriciant

$$
\widetilde{Y}(\eta+k T) \equiv \widetilde{Y}(\eta) Y^{k}(T), \quad k=1,2, \ldots
$$

rewrite the formula for the solution $L(t)$ to $(18)$ as

$$
\begin{aligned}
L(t)= & \int_{0}^{\infty}(\tilde{Y}(\eta))^{*} \tilde{Y}(\eta) d \eta=\sum_{k=0}^{\infty} \int_{k T}^{(k+1) T}(\tilde{Y}(\eta))^{*} \tilde{Y}(\eta) d \eta \\
= & \int_{0}^{T}(\tilde{Y}(\eta))^{*} \tilde{Y}(\eta) d \eta+(Y(T))^{*}\left[\int_{0}^{T}(\tilde{Y}(\eta))^{*} \tilde{Y}(\eta) d \eta\right] Y(T) \\
& +\left(Y^{2}(T)\right)^{*}\left[\int_{0}^{T}(\tilde{Y}(\eta))^{*} \tilde{Y}(\eta) d \eta\right] Y^{2}(T)+\cdots
\end{aligned}
$$

Denote $D=\int_{0}^{T}(\tilde{Y}(\eta))^{*} \widetilde{Y}(\eta) d \eta$. Then we obviously obtain the inequality

$$
\|L(t)\| \leq\|D\|+\|Y(T)\|^{2}\|D\|+\|Y(T)\|^{4}\|D\|+\cdots .
$$

By (10) and (15), we infer $\|Y(T)\|^{2}<1$. Consequently,

$$
\|L(t)\| \leq \frac{1}{1-\|Y(T)\|^{2}}\|D\|
$$

Using the definition of $D$ and (19), we have

$$
\begin{aligned}
\|D\| & \leq \int_{0}^{T}\|\tilde{Y}(\eta)\|^{2} d \eta \leq\left(v_{\max }\right)^{N} \int_{0}^{T} \exp \left(-\frac{\mu \eta}{2 H_{\max }}\right) d \eta \\
& =\frac{2 H_{\max }\left(v_{\max }\right)^{N}}{\mu}\left(1-\exp \left(-\frac{\mu T}{2 H_{\max }}\right)\right) \leq \frac{2 H_{\max }\left(v_{\max }\right)^{N}}{\mu} .
\end{aligned}
$$

Hence, by (15), we obtain

$$
\|L(t)\| \leq \frac{2 H_{\max }\left(v_{\max }\right)^{N}}{\mu}\left(1-\left(v_{\max }\right)^{N} \exp \left(-\frac{\mu T}{2 H_{\max }}\right)\right)^{-1} .
$$

We now take $K(s)=\frac{1}{2} e^{-\alpha s} I$. Then verification of (17) for the matrices $L(t)$ and $K(s)$ reduces to verification of the inequality

$$
2 e^{\alpha \tau} L(t) B(t) B^{*}(t) L(t)-\frac{1}{2} I<0 .
$$


From (20) we derive

$$
\begin{aligned}
\left\|2 e^{\alpha \tau} L(t) B(t) B^{*}(t) L(t)\right\| \leq & 2 e^{\alpha \tau}\|L(t)\|^{2}\|B(t)\|^{2} \\
\leq & \frac{8 e^{\alpha \tau}\left(H_{\max }\right)^{2}\left(v_{\max }\right)^{2 N}}{\mu^{2}} \\
& \times\left(1-\left(v_{\max }\right)^{N} \exp \left(-\frac{\mu T}{2 H_{\max }}\right)\right)^{-2}\|B(t)\|^{2} .
\end{aligned}
$$

Hence, for every continuous matrix $B(t)$ and every $\tau>0$, if $\mu>\mu_{*}$, where $\mu_{*}$ is given by (16), then

$$
\left\|2 e^{\alpha \tau} L(t) B(t) B^{*}(t) L(t)\right\|<\frac{1}{2}
$$

i.e., (21) is valid. Consequently, the zero solution to (1) is asymptotically stable.

Theorem 4 is proved.

Remark 3 It follows from the proof that all solutions to (1) tend to zero as $t \rightarrow \infty$.

\section{Conclusion}

In the present paper we considered the systems of delay differential equations with periodic coefficients of the form (1). We established the conditions on the coefficients and the parameter under which the zero solution is asymptotically stable. These conditions are formulated in terms of inequalities. This result allows us to study the asymptotic stability of the zero solution to time-delay systems of such type without spectral methods (analogs of the Lyapunov-Floquet theory) and Lyapunov-Krasovskii functionals. In this connection let us note $[24,25]$ that the stability of solutions to nonautonomous linear delay differential systems has been studied by using the approaches based on the so-called Azbelev $W$-transform and the Bohl-Perron type theorem, respectively. Using the results of $[16,17]$, it is easy to write down the estimates for solutions to (1) characterizing the exponential decay rate at infinity.

It should be noted that the results obtained in the present paper can be extended to the systems with several delays of the form

$$
\frac{d}{d t} y(t)=\mu A(t) y(t)+\sum_{j=1}^{m} B_{j}(t) y\left(t-\tau_{j}\right), \quad t>0,
$$

where $\tau_{j}>0, j=1, \ldots, m$. Indeed, if there are matrices $L(t)$ and $K_{j}(s)$ satisfying (6), (7), and the Riccati type matrix differential inequality of the form

$$
\begin{aligned}
& \frac{d}{d t} L(t)+\mu L(t) A(t)+\mu A^{*}(t) L(t) \\
& \quad+\sum_{j=1}^{m} L(t) B_{j}(t) K_{j}^{-1}\left(\tau_{j}\right) B_{j}^{*}(t) L(t)+\sum_{j=1}^{m} K_{j}(0)<0,
\end{aligned}
$$


then the zero solution to (22) is asymptotically stable. This inequality is an analog of (17). As above, we may take the solution to (18) as $L(t)$ and

$$
K_{j}(s)=\gamma_{j} e^{-\alpha_{j} s} I, \quad \alpha_{j}>0, \gamma_{j}>0, \sum_{j=1}^{m} \gamma_{j}<1
$$

Then verification of (23) reduces to verification of the inequality

$$
\sum_{j=1}^{m} \frac{1}{\gamma_{j}} e^{\alpha_{j} \tau_{j}} L(t) B_{j}(t) B_{j}^{*}(t) L(t)-\left(1-\sum_{j=1}^{m} \gamma_{j}\right) I<0 .
$$

This inequality is an analog of (21). By (20), it gives us immediately the condition for $\mu$ under which the zero solution to (22) is asymptotically stable.

\section{Competing interests}

The authors declare that they have no competing interests.

\section{Authors' contributions}

All authors contributed equally to the writing of this paper. All authors read and approved the final manuscript.

\section{Acknowledgements}

The authors were supported by the Russian Foundation for Basic Research (project no. 13-01-00329). The authors are grateful to the anonymous referees for the helpful comments and suggestions.

Received: 13 April 2015 Accepted: 25 September 2015 Published online: 13 October 2015

\section{References}

1. El'sgol'ts, LE, Norkin, SB: Introduction to the Theory and Application of Differential Equations with Deviating Arguments. Academic Press, New York (1973)

2. Hale, JK: Theory of Functional Differential Equations. Springer, New York (1977)

3. Korenevskii, DG: Stability of Dynamical Systems Under Random Perturbations of Parameters: Algebraic Criteria. Naukova Dumka, Kiev (1989)

4. Györi, I, Ladas, G: Oscillation Theory of Delay Differential Equations: With Applications. Oxford Mathematical Monographs. Oxford Science Publications. Clarendon, Oxford (1991)

5. Khusainov, D, Shatyrko, AV: The Method of Lyapunov Functions in the Investigation of the Stability of Functional Differential Systems. Kiev University Press, Kiev (1997)

6. Kolmanovskii, VB, Myshkis, AD: Introduction to the Theory and Applications of Functional Differential Equations. Mathematics and Its Applications, vol. 463. Kluwer Academic, Dordrecht (1999)

7. Gu, K, Kharitonov, VL, Chen, J: Stability of Time-Delay Systems. Control Engineering. Birkhäuser, Boston (2003)

8. Azbelev, NV, Maksimov, VP, Rakhmatullina, LF: Introduction to the Theory of Functional Differential Equations: Methods and Applications. Contemporary Mathematics and Its Applications, vol. 3. Hindawi Publishing Corporation, Cairo (2007)

9. Michiels, W, Niculescu, SI: Stability and Stabilization of Time-Delay Systems: An Eigenvalue-Based Approach. Advances in Design and Control, vol. 12. SIAM, Philadelphia (2007)

10. Agarwal, RP, Berezansky, L, Braverman, E, Domoshnitsky, A: Nonoscillation Theory of Functional Differential Equations with Applications. Springer, New York (2012)

11. Kharitonov, VL: Time-Delay Systems: Lyapunov Functionals and Matrices. Control Engineering. Springer, New York (2013)

12. MacDonald, N: Biological Delay Systems: Linear Stability Theory. Cambridge Studies in Mathematical Biology, vol. 8. Cambridge University Press, Cambridge (1989)

13. Kuang, Y: Delay Differential Equations with Applications in Population Dynamics. Mathematics in Science and Engineering, vol. 191. Academic Press, Boston (1993)

14. Erneux, T: Applied Delay Differential Equations. Surveys and Tutorials in the Applied Mathematical Sciences, vol. 3. Springer, New York (2009)

15. Demidenko, GV, Matveeva, II: On stability of solutions to linear systems with periodic coefficients. Sib. Math. J. 42, 282-296 (2001)

16. Demidenko, GV, Matveeva, II: Asymptotic properties of solutions to delay differential equations. Vestn. Novosib. Gos. Univ. Ser. Mat. Mekh. Inform. 5, 20-28 (2005)

17. Demidenko, GV, Matveeva, II: Stability of solutions to delay differential equations with periodic coefficients of linear terms. Sib. Math. J. 48, 824-836 (2007)

18. Demidenko, GV, Matveeva, II: On stability of solutions to quasilinear periodic systems of differential equations. Sib. Math. J. 45, 1041-1052 (2004)

19. Matveeva, II: Estimates of solutions to a class of systems of nonlinear delay differential equations. J. Appl. Ind. Math. 7 557-566 (2013) 
20. Demidenko, GV, Matveeva, II: On estimates of solutions to systems of differential equations of neutral type with periodic coefficients. Sib. Math. J. 55, 866-881 (2014)

21. Bylov, BF, Vinograd, RE, Grobman, DM, Nemytskii, W: Theory of Lyapunov Exponents and Its Applications to Problems of Stability. Nauka, Moscow (1966)

22. Daleckii, J, Krein, MG: Stability of Solutions of Differential Equations in Banach Space. Translations of Mathematical Monographs, vol. 43. Am. Math. Soc., Providence (1974)

23. Hartman, P: Ordinary Differential Equations. SIAM, Philadelphia (2002)

24. Domoshnitsky, A, Gitman, M, Shklyar, R: Stability and estimate of solution to uncertain neutral delay systems. Bound. Value Probl. 2014, 55 (2014)

25. Berezansky, L, Diblík, J, Svoboda, Z, Šmarda, Z: Simple uniform exponential stability conditions for a system of linear delay differential equations. Appl. Math. Comput. 250, 605-614 (2015)

\section{Submit your manuscript to a SpringerOpen ${ }^{\circ}$} journal and benefit from:

- Convenient online submission

Rigorous peer review

- Immediate publication on acceptance

- Open access: articles freely available online

- High visibility within the field

- Retaining the copyright to your article 